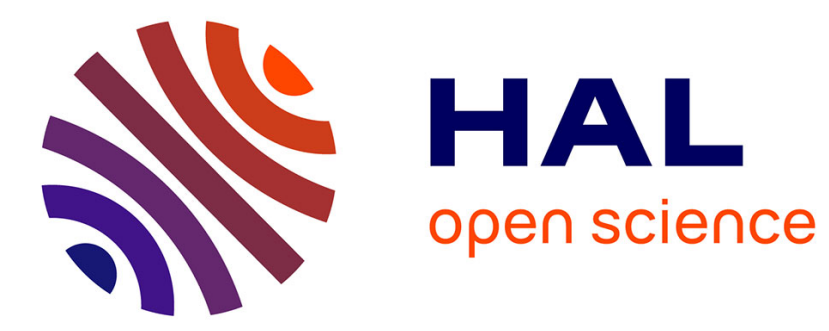

\title{
Temperature and Polarization Dependent XANES Measurements on Single Crystal PbTiO3
}

\author{
B. Ravel, E. Stern
}

\section{To cite this version:}

B. Ravel, E. Stern. Temperature and Polarization Dependent XANES Measurements on Single Crystal PbTiO3. Journal de Physique IV Proceedings, 1997, 7 (C2), pp.C2-1223-C2-1224. 10.1051/jp4:19972204 . jpa-00255276

\section{HAL Id: jpa-00255276 https://hal.science/jpa-00255276}

Submitted on 1 Jan 1997

HAL is a multi-disciplinary open access archive for the deposit and dissemination of scientific research documents, whether they are published or not. The documents may come from teaching and research institutions in France or abroad, or from public or private research centers.
L'archive ouverte pluridisciplinaire HAL, est destinée au dépôt et à la diffusion de documents scientifiques de niveau recherche, publiés ou non, émanant des établissements d'enseignement et de recherche français ou étrangers, des laboratoires publics ou privés. 


\title{
Temperature and Polarization Dependent XANES Measurements on Single Crystal $\mathrm{PbTiO}_{3}$
}

\author{
B. Ravel and E.A. Stern \\ Department of Physics, Box 351560, University of Washington, Seattle, WA 98195, U.S.A.
}

\begin{abstract}
We have measured the X-ray Absorption Near Edge Structure (XANES) of single crystal $\mathrm{PbTiO}_{3}$ as a function of temperature and polarization throughout its tetragonal phase. The broken centrosymmetry at the Ti site gives rise to a distinctive peak in the region before the main rising edge. The strong polarization dependence of this peak is consistent with the displacement of the Ti atom from a site of centrosymmetry along the $c$-axis. We find that the dependence upon temperature and polarization of the area under this peak is consistent with the presence of an order-disorder component in the dynamics of this system as it approaches its phase boundary and that the disordering can be ascertained from the XANES with greater accuracy than from the EXAFS alone.
\end{abstract}

At $763 \mathrm{~K}, \mathrm{PbTiO}_{3}$ undergoes a ferroelectric to paraelectric transition. Simultaneously, its crystallographic structure changes from tetragonal and non-centrosymmetric to cubic and centrosymmetric. A displacive model has been suggested[1] for this transition, wherein the tetragonal elongation and the broken centrosymmetry relax into the cubic structure above the phase transition. In previous work[2], we investigated the phase transition in $\mathrm{PbTiO}_{3}$ using Extended X-ray Absorption Fine Structure (EXAFS) spectroscopy. Our EXAFS analysis showed persistent local disorder about both the titanium and lead sites, indicating the presence of an order-disorder component to this transition. We have confirmed and refined these EXAFS results with improved measurements of the Ti $K$ edge EXAFS, which agree with previous EXAFS measurements[2] below and above the phase transition, and with new temperature and polarization dependent X-ray Absorption Near Edge Structure (XANES) measurements, which we present here.

\section{Experimental Details}

Our XANES measurements were performed on a single crystal of $\mathrm{PbTiO}_{3}$ at beamline X11a at the National Synchrotron Light Source. We examined the crystal under crossed polarizers to find a $700 \mu \times 1000 \mu$ monodomain region with the crystallographic $c$-axis in the plane of the crystal's surface. Fluorescence measurements were obtained with the $c$ axis perpendicular to the electric vector of the incoming photons, yielding $a$-oriented data, and at an angle of $50.6^{\circ}$ relative to the electric vector, from which we extracted $c$-oriented data. In both orientations, the crystal was mounted on a furnace and XANES data were obtained in the temperature range $300 \mathrm{~K}$ to $730 \mathrm{~K}$. All EXAFS data referenced in this paper were obtained in transmission on polycrystalline samples at the same beamline.

\section{XANES Analysis}

We have shown[3] and theoretical calculations have confirmed[4] that a pre-edge peak in transition metal oxide materials arises from broken centrosymmetry in the local structure about the transition metal site, driving the hybridization of empty $\mathrm{Ti} d$ states with $\mathrm{O} p$ states, and allowing the otherwise forbidden dipole transition of the Ti $s$ electron into its unoccupied, hybridized $d$ states. From a perturbation theory argument and as verified by calculation[4], one expects the integrated area under that peak to be proportional to the square of a distortion parameter describing the magnitude of the broken centrosymmetry, $\mathcal{A}=\gamma d^{2}$, where $\gamma$ is the proportionality constant. In $\mathrm{PbTiO}_{3}$ the distortion is entirely along the $c$ axis, thus the Ti-O bond lengths in the $\mathrm{O}$ octahedron are split into three distances - 4 equidistant planar bond lengths and two axial, one of which is longer than the planar bonds and the other shorter. The parameter describing the distortion is the distance of the Ti from the midpoint between the axial oxygens, $d=\left(r_{\text {long }}-r_{\text {short }}\right) / 2$. From our EXAFS results we obtained the temperature dependence of $d$ as well as of the local unit cell dimensions, which remain constant with temperature. These results differ from the diffraction, which find a displacive transition to a cubic structure. [5]

Fig. (2) shows the polarized XANES of $\mathrm{PbTiO}_{3}$ at three temperatures in the ferroelectric, tetragonal phase. The size of the peak in the $c$ orientation diminishes with temperature and the small peak in the $a$ orientation increases. From our EXAFS measurements we find that the local distortions are larger than those inferred by neutron diffraction[5]. This is explained by a disordering model wherein small regions of the crystal randomly rotate by $90^{\circ}$. As more of these regions in the crystal randomly rotate to orientations perpendicular to the incoming photons, the size of the c-oriented peak diminishes and the a-oriented peak increases. Relating areas under peaks to the distortion parameter 
measured by EXAFS we obtain $\gamma=12.5 \pm 0.8$. For comparison, we obtain values for $d$ and $d$ from EXAFS and XANES measurements on $\mathrm{BaTiO}_{3}$. We find that $\gamma$ is about 15 for this material.

A purely displacive model would show the same qualitative behavior of the $a$ - and $c$-oriented peaks. Should the magnitude of the distortion displacively diminish, the area of the $c$-oriented peak would also diminish. The growth of the $a$-oriented peak could then be explained by the growth of a thermally averaged distortion parameter (TADP) due to the uncorrelated root mean squared displacements (RMSD) of the planar O bonds. In EuTiO $\mathrm{E}_{3}$, cubic perovskite with no static local distortion, a tiny pre edge peak is observed which grows with temperature. The RMSD of the $O$ bonds was measured from the Ti $K$ edge EXAFS on this material at four temperatures between $15 \mathrm{~K}$ and $500 \mathrm{~K}$ and found to be consistent with an Einstein temperature of $479 \pm 14 \mathrm{~K}$. It can be shown that the square of the TADP is half of the RMSD. Since the TADP is a small perturbation, $\mathcal{A}=\gamma d_{t}^{2}$, as before, where $d_{t}$ is the TADP and $\gamma=14.5 \pm 1.7$.

From our EXAFS analysis, we obtained the temperature dependence of the TADP of the planar $O$ bonds in $\mathrm{PbTiO}_{3}$. We can relate this to the peak areas in the $a$ orientation. Since we have found $\gamma$ to be roughly constant for Ti-O bonds in several similar materials, we would expect the same $\gamma$ to predict the peak sizes in the $a$-oriented measurements if those peaks are due solely to thermal motion, as predicted by a displacive model. Using this TADP and a $\gamma$ of about 14, we predict areas only half of those found in the data which is outside the uncertainty of the prediction, as shown in Fig. (2.)

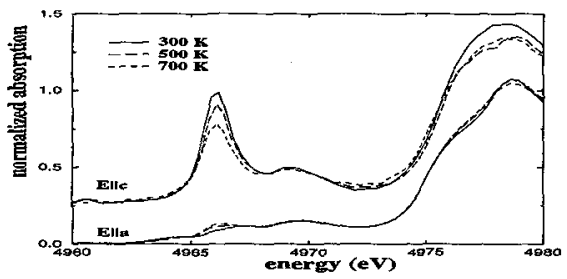

Figure 1. Orientation and temperature dependence of XANES in $\mathrm{PbTiO}_{3}$. The c-oriented X.ANES was obtained from our measurements by $\mu_{c}=\left(\mu_{50.6}-\sin ^{2}(50.6) \cdot \mu_{a}\right) / \cos ^{2}(50.6)$.

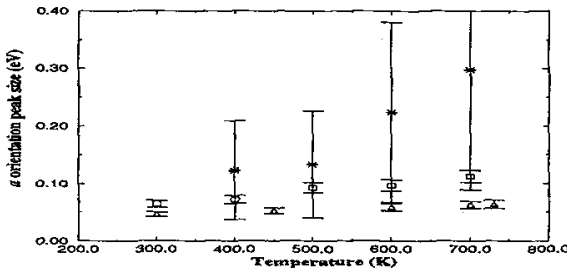

Figure 2. $a$ orientation peak sizes in the ferroelectric phase, the squares are the measured sizes, the triangles are the predicted sizes from the TADP, and the stars are the predicted sizes from the disordering model.

Thus random $90^{\circ}$ rotations of small regions in the $\mathrm{PbTiO}_{3}$ crystal are needed to account for the differences between the measurements of the unit cell dimensions measured by neutron diffraction and by the Ti $K$ edge EXAFS (see Fig. (11) in Ref. [2], our new EXAFS results show the same behavior with smaller uncertainty). For instance at $700 \mathrm{~K}, 60 \mathrm{~K}$ below the phase transition, we find that $38 \%$ of the material is so rotated. From these numbers and our measurements of the areas of the $c$-oriented peaks, we can predict the sizes of the $a$-oriented peaks due to this disordering. From these measurements, we always over-predict the sizes of the $a$-oriented peaks. However, the level of uncertainty in our measurements of the $c$ axis lengths leads to large uncertainties in these predictions. Our data, therefore, are consistent with the disordering model.

\section{Conclusion}

We find that the temperature and orientation dependent XANES of $\mathrm{PbTiO}_{3}$ yields significant information about the nature of this phase transition. We find that we can quantitatively relate the local structure of several Ti perovskites with the pre-edge peak in their XANES. With this relationship, we find that the XANES of the $a$-oriented PbTiO single crystal is inconsistent with a purely displacive model and can be explained within the context of a phase transition with a significant order-disorder component.

\section{Acknowledgments}

We thank D. Haskel, S. Kelly, A. Frenkel, and D. Brewe for their assistance collecting the data and Y. Yacoby for useful discussion. This research was supported by D.O.E. grant \#DE-FG06-90ER45425.

\section{References}

[1] Burnes G. and Scott B.A. Phys. Rev. B7 (1973) 3088-3101.

[2] Sicron N., Ravel B., Yacoby, Y. Stern E.A., and Dogan, F, Phys. Rev B50 (1994) 13168-131180.

[3] Ravel B. and Stern E.A. Physica B 208\&209 (1995) 316-318

[4] Vedrinskii R.V. this proceedings

[5] Nelmes R.J., Plitz R.O., Kuhs, W.F., Tun, Z. and Restori, R., Ferroelectrics 108 (1990) 165-170 